Bull. Korean Math. Soc. 48 (2011), No. 6, pp. 1261-1270

http://dx.doi.org/10.4134/BKMS.2011.48.6.1261

\title{
CONTINUITY OF SPECTRA AND COMPACT PERTURBATIONS
}

\author{
Salvador Sánchez-Perales and Slaviša V. Djordjević
}

\begin{abstract}
In this note we give conditions for continuity of spectrum approximative point spectrum and defect spectrum on the set $\{T\}+\mathcal{K}(X)$, where $T \in \mathcal{B}(X)$ and $\mathcal{K}(X)$ is the set of compact operators.
\end{abstract}

\section{Introduction}

Let $X$ be a Banach space, and let $\mathcal{B}(X)$ denote the algebra of bounded linear operators defined on $X$ into itself. For $T \in \mathcal{B}(X)$, let $\sigma(T), \sigma_{e}(T), \sigma_{l e}(T)$, $\sigma_{r e}(T), \sigma_{a}(T)$ and $\sigma_{s}(T)$ denote respectively the spectrum, the essential spectrum, the left essential spectrum, the right essential spectrum, the approximate point spectrum and the surjective spectrum. We set $\sigma_{l r e}(T)=\sigma_{l e}(T) \cap \sigma_{r e}(T)$. Let $\mathcal{S}$ denote the collection of all non-empty compact subsets of $\mathbb{C}$. Equipping $\mathcal{S}$ with the Hausdorff metric, the spectrum can be viewed as a function $\sigma: \mathcal{B}(X) \rightarrow \mathcal{S}$ mapping operators $T \in \mathcal{B}(X)$ into their spectrum $\sigma(T)$. In general the spectrum is not continuous, but it is always upper semi-continuous (see [8]).

Given an operator $T \in \mathcal{B}(X)$, we write $N(T)$ and $R(T)$ for null space and range of $T$. Denote $\alpha(T)=\operatorname{dim} N(T)$ and $\beta(T)=\operatorname{dim} X / R(T)$. An operator $T$ is called upper semi-Fredholm $\left(T \in \Phi_{+}(X)\right)$, respectively lower semi-Fredholm $\left(T \in \Phi_{-}(X)\right)$, if $R(T)$ is closed and $\alpha(T)<\infty$, respectively $\beta(T)<\infty$. If $T$ is either upper or lower semi-Fredholm, then $T$ is called a semi-Fredholm operator $\left(T \in \Phi_{ \pm}(X)\right)$. When $T$ is both upper and lower semi-Fredholm, $T$ is a Fredholm operator $(T \in \Phi(X))$. The index of a semi-Fredholm operator $T$ is defined as $i(T)=\alpha(T)-\beta(T)$. We set $\Phi_{+}^{-}(X)=\{T \in \mathcal{B}(X) \mid T \in$ $\Phi_{+}(X)$ and $\left.i(T) \leq 0\right\}, \Phi_{-}^{+}(X)=\left\{T \in \mathcal{B}(X) \mid T \in \Phi_{-}(X)\right.$ and $\left.i(T) \geq 0\right\}$ and $\Phi_{0}(X)=\{T \in \Phi(X) \mid i(T)=0\}$.

The ascent of $T \in \mathcal{B}(X)$, denoted by $\operatorname{asc}(T)$, and the descent of $T$, denoted by $\operatorname{des}(T)$, are respectively the least non-negative integer $n$ such that $N\left(T^{n}\right)=$ $N\left(T^{n+1}\right)$, respectively $R\left(T^{n}\right)=R\left(T^{n+1}\right)$. If no such $n$ exists, then $\operatorname{asc}(T)=$

Received July 20, 2010; Revised October 26, 2010.

2010 Mathematics Subject Classification. 47A10, 47 A53.

Key words and phrases. The continuity of the spectrum.

(C)2011 The Korean Mathematical Society 
$\infty$, respectively $\operatorname{des}(T)=\infty$. We say that $T$ has finite ascent (resp. descent) at $\lambda \in \mathbb{C}$ if $\operatorname{asc}(\lambda-T)<\infty$ (resp. $\operatorname{des}(\lambda-T)<\infty)$.

The Weyl spectrum, the Browder spectrum, the point spectrum and the set of Riesz points of $T \in \mathcal{B}(X)$ are defined respectively by $\sigma_{w}(T)=\{\lambda \in$ $\left.\mathbb{C} \mid \lambda-T \notin \Phi_{0}(X)\right\}, \sigma_{b}(T)=\left\{\lambda \in \mathbb{C} \mid \lambda-T \notin \Phi_{0}(X)\right.$ or $\left.\operatorname{asc}(\lambda-T)=\infty\right\}$, $\sigma_{p}(T)=\{\lambda \in \mathbb{C} \mid \lambda$ is an eigenvalue of $T\}$ and $\pi_{0}(T)=\{\lambda \in \mathbb{C} \mid \lambda$ is a pole of $T$ of finite rank $\}$.

Let $T_{n}, T \in \mathcal{B}(X)$. We say that $T_{n}$ converges in norm to $T$, denoted by $T_{n} \rightarrow T$, if $\lim _{n \rightarrow \infty}\left\|T_{n}-T\right\|=0$. A function $\tau$, defined on $\mathcal{B}(X)$, whose values are non-empty compact subsets of $\mathbb{C}$ is said to be upper (lower) semicontinuous at $T$, if $T_{n} \rightarrow T$ implies $\lim \sup \tau\left(T_{n}\right) \subseteq \tau(T)\left(\tau(T) \subseteq \liminf \tau\left(T_{n}\right)\right)$. It is known that if $\tau$ is bounded on convergent sequences, then $\tau$ is continuous in "the Hausdorff metric" if and only if $\tau$ is both upper and lower semi-continuous at $T$.

The rest of this paper is organized as follows. In Section 2, we present relations between Browder's type theorems and continuity of different spectrums. In Section 3, we give conditions for continuity of spectrum when restricted to $\{T\}+\mathcal{K}(X)$ where $T \in \mathcal{B}(X)$ and $\mathcal{K}(X)$ is the set of compact operators. And in Section 4, we assume continuity of the spectrum at $T \in \mathcal{B}(X)$ and give sufficient conditions for continuity of spectrum at $T+K$, where $K \in \mathcal{K}(X)$.

\section{The Browder's theorem and continuity of spectrum}

We say that Browder's theorem holds for $T \in \mathcal{B}(X)$ if

$$
\sigma(T) \backslash \sigma_{w}(T)=\pi_{0}(T)
$$

(or equivalently $\sigma_{b}(T)=\sigma_{w}(T)$ ).

A bounded linear operator $T \in \mathcal{B}(X)$ is said to have the single valued extension property (SVEP, for short) at $\lambda \in \mathbb{C}$, if for every open neighborhood $U_{\lambda}$ of $\lambda$, the only analytic function $f: U_{\lambda} \rightarrow X$ which satisfies the equation $(T-\mu) f(\mu)=0$ for all $\mu \in U_{\lambda}$ is the function $f \equiv 0$. Evidently $T$ has SVEP at points in the resolvent $\rho(T)=\mathbb{C} \backslash \sigma(T)$, furthermore by the identity theorem for analytic functions both $T$ and $T^{*}$ have SVEP at every point of the boundary $\partial \sigma(T)$ of the spectrum. In particular, both $T$ and $T^{*}$ have SVEP at the isolated points of $\sigma(T)$.

Proposition 2.1. If $T \in \mathcal{B}(X)$, then

$$
\sigma(T) \backslash \sigma_{w}(T)=\pi_{0}(T) \cup \operatorname{int}\left[\sigma(T) \backslash \sigma_{w}(T)\right] .
$$

Proof. Evidently, $\operatorname{int}\left[\sigma(T) \backslash \sigma_{w}(T)\right] \subseteq \sigma(T) \backslash \sigma_{w}(T)$. Since $\lambda \in \pi_{0}(T) \Longleftrightarrow$ $\operatorname{asc}(\lambda-T)=\operatorname{des}(\lambda-T)<\infty, \alpha(\lambda-T)<\infty \Longleftrightarrow \operatorname{asc}(\lambda-T)=\operatorname{des}(\lambda-T)<\infty$, $\alpha(\lambda-T)=\beta(\lambda-T)<\infty\left[1\right.$, Theorem 3.4], $\lambda \in \sigma(T) \backslash \sigma_{w}(T)$. Hence $\pi_{0}(T) \cup \operatorname{int}\left[\sigma(T) \backslash \sigma_{w}(T)\right] \subseteq \sigma(T) \backslash \sigma_{w}(T)$.

For the opposite inclusion, let $\lambda \in \sigma(T) \backslash \sigma_{w}(T)$. If $\lambda \notin \operatorname{int} \sigma(T)$, then $\lambda \in \partial \sigma(T)$ and $\lambda-T \in \Phi(X)$. Since $T$ and $T^{*}$ have SVEP at $\lambda$, it follows by 
Theorem 3.16 and Theorem 3.17 of [1] that $\operatorname{asc}(\lambda-T)<\infty$ and $\operatorname{des}(\lambda-T)<\infty$. Thus $\lambda \in \pi_{0}(T)$. Now, if $\lambda \in \operatorname{int} \sigma(T)$, then $\lambda \in \operatorname{int}\left[\sigma(T) \backslash \sigma_{w}(T)\right]$, because $\lambda \in \mathbb{C} \backslash \sigma_{w}(T)$ and this set is open.

Given $T \in \mathcal{B}(X)$, define

$$
\begin{aligned}
& \phi_{+}(T)=\left\{\lambda \in \mathbb{C} \mid \lambda-T \in \Phi_{ \pm}(X), N(\lambda-T)\right. \text { is complemented } \\
& \text { and } i(\lambda-T)>0\} \text {, } \\
& \phi_{-}(T)=\left\{\lambda \in \mathbb{C} \mid \lambda-T \in \Phi_{ \pm}(X), R(\lambda-T)\right. \text { is complemented } \\
& \text { and } i(\lambda-T)<0\} \text {. }
\end{aligned}
$$

We set $\phi_{ \pm}(T)=\phi_{+}(T) \cup \phi_{-}(T)$. It is not difficult to prove that all these sets are open.

Let $H$ be a Hilbert space, and let $A \in \mathcal{B}(H)$ be a continuity point of $\sigma$. We know ([4, Theorem 3.1]) that for each $\lambda \in \sigma(A) \backslash \overline{\phi_{ \pm}(A)}$ and $\epsilon>0$, the ball $B(\lambda, \epsilon)$ contains a component of $\pi_{0}(A) \cup \sigma_{\text {lre }}(A)$. Thus, since $\sigma(A) \backslash \sigma_{w}(A) \subseteq$ $\sigma(A) \backslash \overline{\phi_{ \pm}(A)}$ and $\operatorname{int}\left[\sigma(A) \backslash \sigma_{w}(A)\right] \cap\left[\pi_{0}(A) \cup \sigma_{\text {lre }}(A)\right]=\emptyset$, it follows that $\operatorname{int}\left[\sigma(A) \backslash \sigma_{w}(A)\right]=\emptyset([4$, Corollary 3.2]).

Theorem 2.2. Let $H$ be a Hilbert space. If $\sigma$ is continuous at $T \in \mathcal{B}(H)$, then $T$ satisfies Browder's theorem.

Proof. By Proposition 2.1, $\sigma(T) \backslash \sigma_{w}(T)=\pi_{0}(T) \cup \operatorname{int}\left[\sigma(T) \backslash \sigma_{w}(T)\right]$, and by the argument above, $\operatorname{int}\left[\sigma(T) \backslash \sigma_{w}(T)\right]=\emptyset$. Thus $\sigma(T) \backslash \sigma_{w}(T)=\pi_{0}(T)$, i.e., $T$ satisfies Browder's theorem.

For approximate point spectrum we also have an analogous theorem to the previous, see Theorem 2.4.

Proposition 2.3. Let $\lambda \in \sigma(T) \backslash \sigma_{a}(T)$. Then for every sequence of operators $\left\{T_{n}\right\}$ in $\mathcal{B}(X)$ that converges in norm to $T, \lambda \in \liminf \sigma\left(T_{n}\right)$.

Proof. Let $\lambda \in \sigma(T) \backslash \sigma_{a}(T)$ and suppose that for some sequence of operators $\left\{T_{n}\right\}$ that converges in norm to $T, \lambda \notin \liminf \sigma\left(T_{n}\right)$. We may assume without loss of generality that $\lambda \notin \sigma\left(T_{n}\right)$ for every positive integer $n$. Thus, $\lambda-T \in$ $\Phi_{+}(X)$ and $\lambda-T_{n}$ are Fredholm operators with index zero such that $\lambda-T_{n} \rightarrow$ $\lambda-T$. By the continuity of the index, it follows that $i(\lambda-T)=0$. Since already $\lambda-T$ is left invertible, $\lambda-T$ is invertible.

For $T \in \mathcal{B}(X)$, let

$$
\sigma_{e a}(T)=\left\{\lambda \in \mathbb{C} \mid \lambda-T \notin \Phi_{+}^{-}(X)\right\}, \quad \sigma_{e s}(T)=\left\{\lambda \in \mathbb{C} \mid \lambda-T \notin \Phi_{-}^{+}(X)\right\}
$$

and

$$
\sigma_{a b}(T)=\left\{\lambda \in \mathbb{C} \mid \lambda-T \notin \Phi_{+}^{-}(X) \text { or } \operatorname{asc}(\lambda-T)=\infty\right\} .
$$

We say that a-Browder's theorem holds for $T$ if $\operatorname{acc} \sigma_{a}(T) \subseteq \sigma_{e a}(T)$. This condition is equivalent, see for example [7, Theorem 8.3.3], to each of following conditions: 
(1) $\sigma_{e a}(T)=\sigma_{a b}(T)$,

(2) $\sigma_{a}(T)=\sigma_{e a}(T) \cup$ iso $\sigma_{a}(T)$.

Theorem 2.4. Let $H$ be a Hilbert space. If $\sigma_{a}$ is continuous at $T \in \mathcal{B}(H)$, then $T$ satisfies a-Browder's theorem.

Proof. It is well known that the continuity of $\sigma_{a}$ at $T$ implies $\phi_{-}(T) \cap \sigma_{p}(T)=\emptyset$ (see [5, Theorem 5.1]). We show that $\sigma_{a}(T)=\sigma_{e a}(T) \cup$ iso $\sigma_{a}(T)$. It is clear that $\sigma_{e a}(T) \cup$ iso $\sigma_{a}(T) \subseteq \sigma_{a}(T)$. Let $\lambda \in \sigma_{a}(T)$. Suppose that $\lambda \notin \sigma_{e a}(T)$, then $\lambda-T \in \Phi_{+}(H)$ and $i(\lambda-T) \leq 0$.

If $i(\lambda-T)<0$, then $\lambda \in \phi_{-}(T)$, and so $\lambda \notin \sigma_{p}(T)$. Therefore $N(\lambda-T)=$ $\{0\}$, consequently since $\lambda \in \sigma_{a}(T)$, it follows that $R(\lambda-T)$ is not closed. But this is a contradiction to $\lambda-T \in \Phi_{+}(H)$. Thus $i(\lambda-T)=0$, which implies that $\lambda \in \sigma(T) \backslash \sigma_{w}(T)$.

On other hand, Proposition 2.3 and continuity of $\sigma_{a}$ at $T$ imply that $\sigma$ is continuous at $T$, and hence by Theorem 2.2, $T$ satisfies Browder's theorem. Thus $\lambda \in \sigma(T) \backslash \sigma_{w}(T)=\pi_{0}(T)$ that implies $\lambda \in$ iso $\sigma_{a}(T)$.

From Theorems 2.2 and 2.4 we can see that there is a connection between Browder's type theorems and continuity of different spectrums. This fact also was put out in Theorem 2.1 and Theorem 2.2 of [6], but the latter with one omission. In fact, Browder's theorem implies the equivalence of continuity of Weyl spectrum and Browder spectrum, but not the equivalence of continuity of the (usual) spectrum and the Weyl spectrum. To see this, review Example 4.1 and Remark 4.2 of Section 4. In order to have the second equivalence, additional conditions are necessary, see [3, Theorem 14.17].

\section{Continuity of spectrum on $\{T\}+\mathcal{K}(X)$}

It is known that if $\lambda \in$ iso $\sigma(T)$, then for every sequence $\left\{T_{n}\right\}$ in $\mathcal{B}(X)$ that converges in norm to $T, \lambda \in \liminf \sigma\left(T_{n}\right)$ (see [8, Theorem 2]). Moreover, since $\lim \inf \sigma\left(T_{n}\right)$ is a closed set, it follows that iso $\sigma(T) \subset \liminf \sigma\left(T_{n}\right)$. Therefore the spectrum is continuous at every compact operator $K$.

We have observed that the spectrum is continuous on the space of compact operators $\mathcal{K}(X)$. Nevertheless, this does not guarantee that the spectrum is continuous on the set of compact perturbations of an operator $T \in \mathcal{B}(X)$, i.e., on $\{T\}+\mathcal{K}(X)$. In fact, let $U$ be the unilateral shift on $\ell^{2}(\mathbb{N})$ and let $T, K_{n}$ be operators defined on $\ell^{2}(\mathbb{N}) \oplus \ell^{2}(\mathbb{N})$ as

$$
T=\left[\begin{array}{cc}
U & 0 \\
0 & U^{*}
\end{array}\right] \quad K_{n}=\left[\begin{array}{cc}
0 & \frac{1}{n}\left(I-U U^{*}\right) \\
0 & 0
\end{array}\right] .
$$

Then $\left\{K_{n}\right\}_{n \in \mathbb{N}}$ are compact (one-dimensional) operators and $T+K_{n} \rightarrow T+0$, but $\sigma\left(T+K_{n}\right) \not \rightarrow \sigma(T+0)$. Indeed, each $T+K_{n}$ is similar to $T+K_{1}$ and $T+K_{1}$ is an unitary operator, so for every $n, \sigma\left(T+K_{n}\right)=\sigma\left(T+K_{1}\right)=\{\lambda \in \mathbb{C}|| \lambda \mid=1\}$, and $\sigma(T+0)=\{\lambda \in \mathbb{C}|| \lambda \mid \leq 1\}$. 
Let $T, T_{n} \in \mathcal{B}(X)$ be such that $T_{n}$ converge in norm to $T$. It is known that if $\lambda$ is a finite rank pole of $T$, then there exists a finite rank pole $\lambda_{n}$ of $T_{n}$, for all $n$ large, such that $\left\{\lambda_{n}\right\}$ converges to $\lambda$. Hence, we have next lemma:

Lemma 3.1. $\pi_{0}: \mathcal{B}(X) \rightarrow \mathcal{S}$ is lower semi-continuous.

Recall that

$$
\sigma_{b}(T)=\bigcap_{\substack{K \in \mathcal{K}(X) \\ T K=K T}} \sigma(T+K) \quad \text { and } \quad \sigma_{w}(T)=\bigcap_{K \in \mathcal{K}(X)} \sigma(T+K) .
$$

Theorem 3.2. Let $T \in \mathcal{B}(X)$ and $K_{n}, K \in \mathcal{K}(X)$ be such that $K_{n} \rightarrow K$ and $T K=K T$. If $T$ has finite ascent at every $\lambda \in \sigma_{p}(T)$, then $\sigma\left(T+K_{n}\right) \rightarrow$ $\sigma(T+K)$.

Proof. Let us remember that the spectrum $\sigma$ is upper semi-continuous (see [8, Theorem 1]), thus $\lim \sup \sigma\left(T+K_{n}\right) \subseteq \sigma(T+K)$. Hence we only need to prove that $\sigma(T+K) \subseteq \liminf \sigma\left(T+K_{n}\right)$.

Let $\lambda \in \sigma(T+K)$. If $\lambda \in \sigma_{w}(T)$, then by (3.1), $\lambda \in \sigma\left(T+K_{n}\right)$ for all $n \in \mathbb{N}$. Thus $\lambda \in \liminf \sigma\left(T+K_{n}\right)$.

Suppose that $\lambda \notin \sigma_{w}(T)$, this implies that $\lambda-T \in \Phi_{0}(X)$ and consequently $\lambda-(T+K) \in \Phi_{0}(X)$. If $\lambda \notin \sigma(T)$, then $\lambda-T$ is invertible and hence $\operatorname{asc}(\lambda-T)<\infty$, now if $\lambda \in \sigma(T)$, then $\alpha(\lambda-T)>0$, i.e., $\lambda \in \sigma_{p}(T)$, thus from hypothesis $\operatorname{asc}(\lambda-T)<\infty$. Therefore, in any case, $\operatorname{asc}(\lambda-T)<\infty$. Observe that $(\lambda-T)(-K)=-\lambda K+T K=-\lambda K+K T=(-K)(\lambda-T)$. This implies from [1, Theorem 3.43] that $\operatorname{asc}(\lambda-T-K)<\infty$. Thus, since $\operatorname{asc}(\lambda-(T+K))<\infty$ and $\lambda-(T+K) \in \Phi_{0}(X)$, it follows by [1, Theorem 3.4] that $\operatorname{des}(\lambda-(T+K))<\infty$ that implies $\lambda \in \pi_{0}(T+K)$ (see, for example, $[1$, Remark 3.7(c)]). Consequently, by Lemma 3.1,

$$
\lambda \in \liminf \pi_{0}\left(T+K_{n}\right) \subseteq \liminf \sigma\left(T+K_{n}\right) .
$$

It is well known (see for example [7]) that if $T$ or $T^{*}$ has SVEP at every $\lambda \in \sigma(T) \backslash \sigma_{w}(T)$, then Browder's theorem holds for $T$.

Corollary 3.3. If $T \in \mathcal{B}(X)$ has $S V E P$ at any $\lambda \in \sigma(T) \backslash \sigma_{w}(T)$. Then for every $K \in \mathcal{K}(X)$ such that

(i) $T K=K T$ or

(ii) int $\sigma(T+K) \subseteq \sigma(T)$,

it follows that $\sigma\left(T+K_{n}\right) \rightarrow \sigma(T+K)$ for any sequence $\left\{K_{n}\right\} \subset \mathcal{K}(X)$ such that $K_{n} \rightarrow K$.

Proof. By demonstration of Theorem 3.2 we have to observe only the case when $\lambda \in \sigma(T+K) \backslash \sigma_{w}(T)$. By corollary's hypothesis $T$ obeys Browder's theorem, and for $\lambda \in \sigma(T+K) \backslash \sigma_{w}(T)$ we have that $\lambda-T$ is a Fredholm operator of index zero with finite ascent and descent.

(i) If $K \in \mathcal{K}(X)$ commutes with $T$, then by [1, Theorem 3.43] we have that $\lambda-(T+K)$ is a Fredholm operator of index zero with finite ascent and descent, and so $\lambda \in \pi_{0}(T+K) \subset \liminf \sigma\left(T+K_{n}\right)$. 
(ii) If $\lambda \notin \sigma(T)$, then it is clear that $\lambda \notin \operatorname{int} \sigma(T+K)$. Now, if $\lambda \in \sigma(T)$, then $\lambda \in \pi_{0}(T)$ and so $\lambda \notin \operatorname{int} \sigma(T)$. But, since int $\sigma(T+K) \subseteq \operatorname{int} \sigma(T)$, it follows that $\lambda \notin \operatorname{int} \sigma(T+K)$. Thus, in any case $\lambda \notin \operatorname{int} \sigma(T+K)$. This implies that $\lambda \in \partial \sigma(T+K) \backslash \sigma_{e}(T+K) \subseteq \pi_{0}(T+K)$. Hence $\lambda \in \pi_{0}(T+K) \subset$ $\liminf \sigma\left(T+K_{n}\right)$.

Corollary 3.4. Let $H$ be a Hilbert space and let $T \in \mathcal{B}(H)$ be a continuity point of spectrum. If $K$ is a compact operator that commutes with $T$, then $\sigma\left(T+K_{n}\right) \rightarrow \sigma(T+K)$ for any sequence $\left\{K_{n}\right\} \subseteq \mathcal{K}(H)$ that converges to $K$.

Proof. By Theorem 2.2, Browder's theorem holds for $T$, and following the proof of Corollary 3.3, we have that $\sigma\left(T+K_{n}\right) \rightarrow \sigma(T+K)$.

Necessary and sufficient conditions in order that $T$ obeys a-Browder's theorem is $T$ or $T^{*}$ has SVEP at every $\lambda \notin \sigma_{e a}(T)$ ([7, Thm. 8.3.3 and Cor. 8.3.4]). Also, a-Browder's theorem implies Browder's theorem.

It is well known that

$\sigma_{e a}(T)=\bigcap_{K \in \mathcal{K}(X)} \sigma_{a}(T+K)$ and $\sigma_{a b}(T)=\bigcap_{\substack{K \in \mathcal{K}(X) \\ T K=K T}} \sigma_{a}(T+K)($ see $[1$, p. 138] $)$.

Theorem 3.5. Let $K \in \mathcal{K}(X)$ be commutes with $T \in \mathcal{B}(X)$ and let $\left\{K_{n}\right\}$ be a sequence of compacts operators such that $K_{n} \rightarrow K$.

(a) If $T^{*}$ has SVEP at every $\lambda \notin \sigma_{e a}(T)$, then $\sigma_{a}\left(T+K_{n}\right) \rightarrow \sigma_{a}(T+K)$.

(b) If T has SVEP at every $\lambda \notin \sigma_{e s}(T)$, then $\sigma_{s}\left(T+K_{n}\right) \rightarrow \sigma_{s}(T+K)$.

Proof. We will proof only part (a) and by duality we have part (b).

(a) Let $\lambda \in \sigma_{a}(T+K)$. If $\lambda \in \sigma_{e a}(T)$, then $\lambda \in \sigma_{a}(T+F)$ for every $F \in \mathcal{K}(X)$ and consequently $\lambda \in \liminf \sigma_{a}\left(T+K_{n}\right)$.

Suppose that $\lambda \notin \sigma_{e a}(T)$. By theorem's hypothesis, a-Browder's theorem holds for $T$, thus $\lambda \notin \sigma_{a b}(T)$, i.e., $\lambda-T \in \Phi_{+}^{-}(X)$ and $\operatorname{asc}(\lambda-T)<\infty$. Moreover, since $T^{*}$ has SVEP at $\lambda$, it follows that $i(\lambda-T)=0$. From stability of index by compact perturbations and stability of ascent by commutative compact perturbations (for the latter, see proof of Theorem 3.2) we have that $i(\lambda-(T+K))=0$ and $\operatorname{asc}(\lambda-(T+K))<\infty$, so by [1, Theorem 3.4], $\operatorname{des}(\lambda-(T+K))<\infty$. Hence, $\lambda \in \pi_{0}(T+K) \subset \liminf \pi_{0}\left(T+K_{n}\right) \subset$ $\liminf \sigma_{a}\left(T+K_{n}\right)$.

\section{Continuity of spectrum from $T$ to $T+K$}

Question 1. Let $T \in \mathcal{B}(H)$ be a bounded operator such that $\sigma$ is continuous at $T$. Does it follow that $\sigma$ is continuous at $T+K$ for all $K \in K(H)$ ?

Question 2. Let $T \in \mathcal{B}(H)$ be a bounded operator such that $\sigma$ is continuous at $T$. Does it follow that $\sigma$ is continuous at $T+K$ for all $K \in K(H)$ that commutes with $T$ ?

In general context, the answers to these questions are negatives as shown in the following example, which is a modification of [3, Example 14.3(ii)]. 
Example 4.1. Let $\alpha_{n k}=\left(1+\frac{1}{n}\right) \exp \left(2 \pi i \frac{k}{n}\right)$ for all $n \in \mathbb{N}$ and $1 \leq k \leq n$, and consider $M: \ell^{2}(\mathbb{N}) \rightarrow \ell^{2}(\mathbb{N})$ the diagonal operator defined by

$$
M=\left(\begin{array}{cccc}
\alpha_{11} & & & \\
& \alpha_{21} & & \\
& & \alpha_{22} & \\
& & & \ddots
\end{array}\right) .
$$

It is clear that $\sigma_{p}(M)=\left\{\alpha_{n k} \mid n \in \mathbb{N}, 1 \leq k \leq n\right\}$ where each eigenvalue has geometry multiplicity one. Let $\left\{\alpha_{m}\right\}_{m \in \mathbb{N}}$ be an indexation of $\left\{\alpha_{11}, \alpha_{21}\right.$, $\left.\alpha_{22}, \alpha_{31}, \alpha_{32}, \ldots\right\}$. It is not difficult to prove that

$$
\lambda \in \rho(M)=\mathbb{C} \backslash \sigma(M) \Leftrightarrow \inf _{m}\left|\lambda-\alpha_{m}\right|>0 .
$$

Thus

$$
\begin{aligned}
\sigma(M) & =\sigma_{p}(M) \cup\left\{\lambda \in \mathbb{C}\left|\inf _{m}\right| \lambda-\alpha_{m} \mid=0, \text { and for all } m, \lambda \neq \alpha_{m}\right\} \\
& =\left\{\alpha_{m}\right\}_{m \in \mathbb{N}} \cup\{\lambda \in \mathbb{C}|| \lambda \mid=1\} .
\end{aligned}
$$

Let $m \in \mathbb{N}$, since $\inf \left\{\left|\alpha_{m}-\alpha_{j}\right| \mid j \in \mathbb{N}\right.$ and $\left.j \neq m\right\}>0$ and for each $\left\{y_{j}\right\}_{j \in \mathbb{N}} \in \overline{R\left(\alpha_{m}-M\right)}, y_{m}=0$. It follows that $R\left(\alpha_{m}-M\right)$ is a closed subset of $\ell^{2}(\mathbb{N})$. Thus for every $m \in \mathbb{N}, \alpha_{m}$ is an isolated eigenvalue of $M$ and $\alpha_{m}-M$ is a semi-Fredholm operator. Therefore $\left\{\alpha_{m}\right\}_{m \in \mathbb{N}} \subseteq \pi_{0}(M)$.

It is easy to see that $\sigma_{\text {lre }}(M)=\{\lambda \in \mathbb{C}|| \lambda \mid=1\}$. Thus

$$
\phi_{ \pm}(M)=\emptyset, \quad \pi_{0}(M)=\left\{\alpha_{m}\right\}_{m \in \mathbb{N}} \text { and } \sigma_{w}(M)=\{\lambda \in \mathbb{C}|| \lambda \mid=1\} .
$$

From this we obtain automatically by [4, Theorem 3.1] that $\sigma$ is continuous at $M$.

Now, it is clear that $M$ is a normal operator, so by [9, Theorem 4.1], there is a compact operator $K$ that commutes with $T$ and such that $\sigma(M+K)=$ $\sigma_{w}(M)$. Then $\pi_{0}(M+K)=\emptyset$, and also $\phi_{ \pm}(M+K)=\phi_{ \pm}(M)=\emptyset$. Consider $\lambda_{0} \in\{\lambda \in \mathbb{C}|| \lambda \mid=1\}$ and $\epsilon>0$ such that $\{\lambda \in \mathbb{C}|| \lambda \mid=1\} \nsubseteq B\left(\lambda_{0}, \epsilon\right)$. The set $D=\mathbb{C} \backslash B\left(\lambda_{0}, \epsilon\right)$ satisfies that

(i) $\phi_{ \pm}(M+K) \subseteq D$,

(ii) Every component of $\pi_{0}(M+K) \cup \sigma_{\text {lre }}(M+K)=\sigma_{\text {lre }}(M)$ meets $D$.

So by [2, Theorem 3.1], there is a sequence of operators $\left\{A_{n}\right\}_{n \in \mathbb{N}}$ in $\mathcal{B}\left(\ell^{2}(\mathbb{N})\right)$ such that $A_{n} \rightarrow M+K$ and for each $n \in \mathbb{N}, \sigma\left(A_{n}\right) \subseteq D$. Thus, since $\sigma(M+$ $K)=\{\lambda \in \mathbb{C}|| \lambda \mid=1\}$, it follows that

$$
\sigma\left(A_{n}\right) \not \rightarrow \sigma(M+K) .
$$

Conclusion: $\sigma$ is continuous at $M$ and there is a compact operator $K$ that commutes with $M$ such that $\sigma$ is not continuous at $M+K$.

Remark 4.2. The operator $M$ defined in Example 4.1 is not a continuity point of $\sigma_{w}$. Indeed, let $\left\{A_{n}\right\}, K$ and $D$ as in Example 4.1. Then $A_{n}-K \rightarrow M$ and for every natural $n, \sigma\left(A_{n}\right) \subseteq D$. Observe that for each $n \in \mathbb{N}$,

$$
\sigma_{w}\left(A_{n}-K\right)=\sigma_{w}\left(A_{n}\right) \subseteq \sigma\left(A_{n}\right) \subseteq D .
$$


Hence, since $\sigma_{w}(M)=\{\lambda \in \mathbb{C}|| \lambda \mid=1\}$, it follows that $\sigma_{w}\left(A_{n}-K\right) \not \rightarrow$ $\sigma_{w}(M)$, i.e., $\sigma_{w}$ is not continuous at $M$.

In Example 4.1 even when $K$ commutes with $T$ we do not have that $\sigma$ is continuous at $T+K$. Nevertheless if $\sigma$ is restricted on $\{T\}+\mathcal{K}(X)$, then by Corollary 3.4, $\sigma$ is already continuous.

Let $\phi_{+\infty}(T)$ (and $\phi_{-\infty}(T)$ ) be denote respectively the set of $\lambda \in \phi_{+}(T)$ $\left(\lambda \in \phi_{-}(T)\right)$ such that $i(\lambda-T)=\infty(i(\lambda-T)=-\infty)$. We set $\phi_{ \pm \infty}(T)=$ $\phi_{+\infty}(T) \cup \phi_{-\infty}(T)$. With these sets, [5, Lemma 3.1] can be extended to general Banach spaces. In fact:

Lemma 4.3. Let, for $\lambda \notin \overline{\phi_{ \pm \infty}(T)}$, the ball $B(\lambda, \epsilon)$ contains a component of $\sigma_{\text {lre }}(T)$ for any $\epsilon>0$. Then for any sequence $\left\{T_{n}\right\}$ in $\mathcal{B}(X)$ that converges in norm to $T \in \mathcal{B}(X), \lambda \in \liminf \sigma_{\text {lre }}\left(T_{n}\right)$.

Theorem 4.4. If $T \in \mathcal{B}(X)$ is such that for every $\lambda \in \sigma_{\text {lre }}(T) \backslash \overline{\phi_{ \pm}(T)}$ and $\epsilon>0$, the ball $B(\lambda, \epsilon)$ contains a component of $\sigma_{\text {lre }}(T)$, then $\sigma$ is continuous at $T+K$ for all $K \in \mathcal{K}(X)$ such that $T+K$ satisfies Browder's theorem.

Proof. Let $\left\{T_{n}\right\}_{n \in \mathbb{N}}$ be a sequence in $\mathcal{B}(X)$ such that $T_{n} \rightarrow T+K$ and let $\lambda \in \sigma(T+K)$.

If $\lambda \in \sigma_{\text {lre }}(T) \backslash \overline{\phi_{ \pm}(T)}$, then by hypothesis and by Lemma 4.3,

$$
\lambda \in \liminf \sigma_{\text {lre }}\left(T_{n}-K\right) .
$$

Since, for every $n, \sigma_{\text {lre }}\left(T_{n}-K\right)=\sigma_{\text {lre }}\left(T_{n}\right)$, it follows that $\lambda \in \lim \inf \sigma_{\text {lre }}\left(T_{n}\right) \subseteq$ $\lim \inf \sigma\left(T_{n}\right)$.

If $\lambda \in \overline{\phi_{ \pm}(T)}$, then

$$
\lambda \in \overline{\phi_{+}(T)}=\overline{\phi_{+}(T+K)} \subseteq \liminf \sigma_{a}\left(T_{n}\right) \subseteq \liminf \sigma\left(T_{n}\right)
$$

or

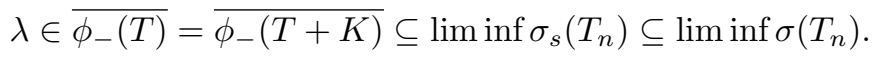

Now suppose that $\lambda \notin \sigma_{\text {lre }}(T) \cup \phi_{ \pm}(T)$. Then $\lambda \notin \sigma_{\text {lre }}(T+K) \cup \phi_{ \pm}(T+K)$, this implies that $\lambda-(T+K) \in \Phi_{0}(X)$ and so $\lambda \in \sigma(T+K) \backslash \sigma_{w}(T+K)$. Since $T+K$ satisfies Browder's theorem it follows that $\lambda \in \pi_{0}(T+K)$. Then, by Lemma 3.1, $\lambda \in \liminf \pi_{0}\left(T_{n}\right) \subseteq \liminf \sigma\left(T_{n}\right)$.

Let $H$ be a Hilbert space, and let $A \in \mathcal{B}(H)$ be a continuity point of $\sigma$. It's not difficult to prove from [4, Theorem 3.1] that if $\lambda \in \sigma_{l r e}(A) \backslash \overline{\phi_{ \pm}(A) \cup \pi_{0}(T)}$, then for every $\epsilon>0$, the ball $B(\lambda, \epsilon)$ contains a component of $\sigma_{\text {lre }}(A)$. With this, we have the following corollary.

Corollary 4.5. Let $H$ be a Hilbert space. If $\sigma$ is continuous at $T \in \mathcal{B}(H)$ and $K$ is a compact operator that commutes with $T$ such that

(i) $\left.\partial \sigma(T+K) \cap \overline{\left[\pi_{0}(T)\right.} \backslash \pi_{0}(T)\right]=\emptyset$

or

(ii) for all $\lambda \in \partial \sigma(T+K) \cap\left[\overline{\pi_{0}(T)} \backslash \pi_{0}(T)\right], \lambda-T$ is semi-Fredholm. Then $\sigma$ is continuous at $T+K$. 
Proof. We show first that $T+K$ satisfies Browder's theorem. By Theorem 2.2 we see that the continuity of $\sigma$ at $T$ implies that $T$ satisfies Browder's theorem. Let $\lambda \in \sigma(T+K) \backslash \sigma_{w}(T+K)$. If $\lambda \in \sigma(T)$, then $\lambda \in \sigma(T) \backslash \sigma_{w}(T)$ and hence $\lambda \in \pi_{0}(T)$, thus $\lambda-T$ is a Weyl operator with finite ascent and descent. When $\lambda \notin \sigma(T)$ this also is true. Thus since $T$ commutes with $K$, it follows that $\lambda-(T+K)$ is a Weyl operator with finite ascent and descent, therefore $\lambda \in \pi_{0}(T+K)$.

Now from an inspection of the proof of Theorem 4.4 we only have to check that $\sigma(T+K) \cap \overline{\pi_{0}(T)} \subseteq \liminf \sigma\left(T_{n}\right)$, where $T_{n} \rightarrow T+K$. Let $\lambda \in \sigma(T+$ $K) \cap \overline{\pi_{0}(T)}$. If $\lambda \in \operatorname{int} \sigma(T+K)$ or $\lambda \in \pi_{0}(T)$, then $\lambda \in \overline{\pi_{0}(T+K)}$ because $T K=K T$. But from Lemma 3.1, $\overline{\pi_{0}(T+K)} \subseteq \liminf \sigma\left(T_{n}\right)$, therefore $\lambda \in$ $\lim \inf \sigma\left(T_{n}\right)$.

Observe that if $\lambda \notin \operatorname{int} \sigma(T+K)$ and $\lambda \notin \pi_{0}(T)$, then $\lambda \in \partial \sigma(T+K) \cap$ $\left[\overline{\pi_{0}(T)} \backslash \pi_{0}(T)\right]$. When hypothesi (i) is satisfied, this case can not happen. Now when hypothesi (ii) is holds, we also have that $\lambda \in \pi_{0}(T+K)$ and so $\lambda \in \liminf \sigma\left(T_{n}\right)$.

Corollary 4.6. Let $H$ be a Hilbert space. If $\sigma$ is continuous at $T \in \mathcal{B}(H)$, then $\sigma$ is continuous at $T+K$ for all $K \in \mathcal{K}(H)$ with

$$
\text { int } \sigma(T+K) \subseteq \sigma(T) \text { and iso } \sigma(T) \subseteq \text { iso } \sigma(T+K) \text {. }
$$

Proof. It is easy to see that int $\left[\sigma(T+K) \backslash \sigma_{w}(T+K)\right]=\emptyset$.

Now, as the previous corollary, $\sigma(T+K) \cap \overline{\pi_{0}(T)} \subseteq \liminf \sigma\left(T_{n}\right)$, where $T_{n} \rightarrow T+K$. Because $\pi_{0}(T) \subseteq \pi_{0}(T+K)$ due to iso $\sigma(T) \subseteq$ iso $\sigma(T+K)$.

\section{References}

[1] P. Aiena, Fredholm and Local Spectral Theory with Applications to Multipliers, Kluwer, 2004.

[2] C. Apostol and B. Morrel, On uniform approximation of operators by simple models, Indiana Univ. Math. J. 26 (1977), no. 3, 427-442.

[3] C. Apostol, L. A. Fialkow, D. A. Herrero, and D. Voiculescu, Approximation of Hilbert Space Operators. Vol. II, Res. Notes Math. 102, Pitman, Boston, 1984.

[4] J. B. Conway and B. B. Morrel, Operators that are points of spectral continuity, Integral Equations Operator Theory 2 (1979), no. 2, 174-198.

[5] _ Operators that are points of spectral continuity. II, Integral Equations Operator Theory 4 (1981), no. 4, 459-503.

[6] S. V. Djordjević and Y. M. Han, Browder's theorems and spectral continuity, Glasg. Math. J. 42 (2000), no. 3, 479-486.

[7] B. P. Duggal, SVEP, Browder and Weyl theorems, Tópicos de Teoría de la Aproximación III, Editores: M.A. Jiménez P., J. Bustamante G. y S.V. Djordjević, Textos Científicos, BUAP, Puebla (2009), 107-146.

[8] J. D. Newburgh, The variation of spectra, Duke Math. J. 18 (1951), 165-176.

[9] C. L. Olsen and J. K. Plastiras, Quasialgebraic operators, compact perturbations, and the essential norm, Michigan Math. J. 21 (1974), 385-397. 
SALVAdor SÁnChez-Perales

Facultad de Ciencias Físico-Matemáticas, BUAP

Río Verde y Av. San Claudio

San Manuel, Puebla, Pue. 72570

MÉXICO

E-mail address: es21254@yahoo.com.mx

Slaviša V. DJordjević

Facultad de Ciencias Físico-Matemáticas, BUAP

Río Verde y Av. San Claudio

San Manuel, Puebla, Pue. 72570

MÉXICO

E-mail address: slavdj@fcfm.buap.mx 